\title{
Euclidean Reconstruction and Auto-Calibration from Continuous Motion
}

\author{
Fredrik Kahl and Anders Heyden * \\ Centre for Mathematical Sciences \\ Lund University \\ Box 118, SE-221 00 Lund, Sweden \\ \{fredrik, andersp\}@maths.lth.se
}

\begin{abstract}
This paper deals with the problem of incorporating natural regularity conditions on the motion in an MAP estimator for structure and motion recovery from uncalibrated image sequences. The purpose of incorporating these constraints is to increase performance and robustness. Autocalibration and structure and motion algorithms are known to have problems with (i) the frequently occurring critical camera motions, (ii) local minima in the non-linear optimization and (iii) the high correlation between different intrinsic and extrinsic parameters of the camera, e.g. the coupling between focal length and camera position. The camera motion (both intrinsic and extrinsic parameters) is modelled as a random walk process, where the inter-frame motions are assumed to be independently normally distributed. The proposed scheme is demonstrated on both simulated and real data showing the increased performance.
\end{abstract}

\section{Introduction}

The problem of estimating both structure and motion from image sequences taken by hand-held video cameras has been treated by many researchers during the past 5 to 10 years, see [8] for a good overview. Significant achievements have been obtained for feature extraction algorithms, tracking methods and reconstruction methods, but also related methods for displaying the results as dense depth maps, mesh generation and texture mapping. The state of the art includes the possibility of making Euclidean reconstructions from basically uncalibrated cameras, using so called auto-calibration techniques, cf. [1, 5, 7, 9, 12, 17, 22, 4, 2].

It has been proven that it is sufficient to know only one intrinsic parameter, e.g. skew zero, in order to obtain a Euclidean reconstruction, see $[10,16]$. This makes it possible to obtain Euclidean reconstructions from uncalibrated

${ }^{*}$ This work has been done within the EU/IST-project 1999-10736, VISIRE and the Swedish Research Council project 95-64-222, JIG. image sequences where the zoom of the camera may vary. However, one problem that is fundamental to all approaches and not dependent on the actual algorithm or implementation of it, is the high correlation between different motion variables. For example, the focal length is usually correlated to the position of the camera, since a change in focal length is similar to a motion towards the scene. Another problem with traditional approaches is that degenerate - or close to degenerate - motions, cf. [20,11], often occur and disturb the bundle adjustment algorithm. Finally, there is always the problem of getting stuck in local minima when a non-linear optimization is adopted. These problems are generic and cannot be solved without using any additional information about the scene geometry or the camera motion.

In the light of the previous drawbacks of the current state-of-the-art algorithms, it is natural to include some smoothness constraints on the intrinsic and extrinsic parameters in order to achieve more stable results. When the image sequence is captured by a hand-held cam-corder with a high frame-rate, the distances between consecutive camera centres are generally small. The actual condition we will use is that the distances between different camera centres are independently and normally distributed, which means that we model the camera motion as a random walk process [19]. A similar model is used for the orientation and intrinsic parameters of the camera. This procedure may easily be generalized to even higher order approximations of smoothness constraints, such as ARMA-models of the motion.

In the present paper we will develop a maximum a posteriori (MAP) estimator, where an a priori probability distribution of the camera motion is utilized. The MAP estimator can be seen as an extension of traditional bundle adjustment, which is in general a maximum likelihood (ML) estimator, cf. [24]. We will show that incorporating these smoothness constraints on the camera motion will give great advantages to conventional methods.

Related Work: The proposed method of using smoothness constraints in the form of a random walk process is related to several previous Kalman filter based approaches 
to the structure from motion problem. In these approaches the Kalman filter estimates both structure and motion parameters as well as their probability densities, e.g. [18, 3]. However, Kalman filtering is a recursive method, i.e. the structure and motion is updated each time a new image is available, while the proposed method in this paper is a batch method, where all measurements are taken into account at the same time. The former approach might be more relevant in a real-time system, while our approach gives more accurate results. A Kalman filter uses a linear update and this is not optimal for the highly non-linear structure and motion problem. Kalman filtering and batch methods for calibrated image sequences using no regularity constraints on the motion were analyzed in [25], and they concluded that the performance difference between these two types of methods is quite large.

In [14] a recursive method is proposed that closely approximates a batch process, using a variable state dimension filter, but still the camera motion is not modelled.

\section{The Camera Model}

The following notations will be used: $\mathbf{X}$ denotes object points in homogeneous coordinates, $\mathbf{x}$ denotes image points in homogeneous coordinates, $\mathbf{x}_{i, j}$ denotes point number $j$ in image $i$. Some further notations will occasionally be needed and will be defined when appropriate.

We will use the standard (uncalibrated) pin-hole camera model:

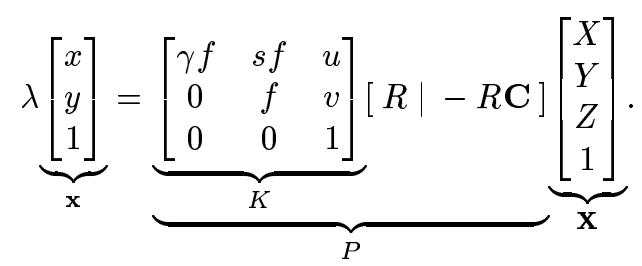

Here, $f$ denotes the focal length, $\gamma$ and $s$ the aspect ratio and the skew and $(u, v)$ the principal point. These are called the intrinsic parameters. Furthermore, $R$ and $\mathbf{C}$ denote the relation between the camera coordinate system and the object coordinate system, where $R$ is a rotation matrix and $\mathbf{C}$ is the position of the camera centre. These are called the extrinsic parameters. When several images of several points are available we will use the notation

$$
\lambda \mathbf{x}_{i, j}=P_{i} \mathbf{X}_{j}, \quad i=1, \ldots m, \quad j=1, \ldots n,
$$

where $m$ denotes the number of images and $n$ denote the number of points.

\section{Overview of the System}

The proposed scheme is embedded in a system to model Euclidean 3D objects and environments from uncalibrated image sequences taken by a continuously moving camera. The complete procedure is briefly summarized below.

1. Extract and track feature image points in the sequence.

2. Estimate projective camera geometry and scene points, using fundamental matrices and trifocal tensors, [21, 6].

2. Upgrade to a Euclidean reconstruction, cf. [16, 22].

3. Apply bundle adjustment, as described in the next sections.

4. Find dense correspondences across the views, triangulate and texture map in order to obtain a 3D model that can be used in a graphics environment, cf. [15, 13].

\section{Modelling of Continuous Motion}

Let us start with the extrinsic camera parameters. The camera centres $\mathbf{C}_{i}, i=1, \ldots, m$, are supposed to follow a continuous trajectory. A reasonable model is then that the centres perform a random walk. More specifically,

$$
\mathbf{C}_{i}-\mathbf{C}_{i+1} \in N\left(0_{3 \times 1}, \sigma_{c} I_{3 \times 3}\right),
$$

where $N(0, \sigma)$ is a normal distribution with zero mean and standard deviation $\sigma$. The sub-indices indicate the dimensions.

The pose of the camera is also supposed to vary smoothly between the imaging instants. Let

$$
B_{1}=\left[\begin{array}{ccc}
0 & 1 & 0 \\
-1 & 0 & 0 \\
0 & 0 & 0
\end{array}\right], \quad B_{2}=\left[\begin{array}{ccc}
0 & 0 & -1 \\
0 & 0 & 0 \\
1 & 0 & 0
\end{array}\right] \text { and } B_{3}=\left[\begin{array}{ccc}
0 & 0 & 0 \\
0 & 0 & 1 \\
0 & -1 & 0
\end{array}\right]
$$

be a base for all skew symmetric $3 \times 3$ matrices. The exponential map of $\sum_{k=1}^{3} \lambda_{k} B_{k}$ is a rotation matrix and the $\lambda_{k}$ are called the Euler angles of the rotation. A natural random walk model for the camera poses $R_{i}, i=1, \ldots, m$ is then

$$
\exp \left(\sum_{k=1}^{3} \lambda_{k} B_{k}\right) R_{i}=R_{i+1}, \quad \lambda_{j} \in N\left(0, \sigma_{a}\right)
$$

It remains to model the intrinsic parameters of the camera. The skew of the camera is often close to zero for ordinary cameras. One way to model this is to assume that the skew is normally distributed with a very low standard deviation. Another option which we choose is to set the skew to zero and use it as a hard constraint. Similarly with the aspect ratio - it is often very close to one, and we fix it to one as a hard constraint.

The focal lengths $f_{i}, i=1, \ldots, m$ vary between imaging instants if the zoom of the camera is used. Since it is the relative changes of focal length that are of interest, a natural choice is

$$
\log \left(f_{i} / f_{i+1}\right) \in N\left(0, \sigma_{f}\right)
$$


The principal points $\left(u_{i}, v_{i}\right), i=1, \ldots, m$ are known to vary when the focal length changes. However, these variations are known to be small so the principal point is located within a small region, often close to the image centre. Let $(\bar{u}, \bar{v})$ denote the mean principal point. A random walk model for the principal points is given by

$$
\left(u_{i}, v_{i}\right)-(\bar{u}, \bar{v}) \in N\left(0_{2 \times 1}, \sigma_{p p} I_{2 \times 2}\right) .
$$

\section{Bundle Adjustment}

A bundle adjustment algorithm was developed for estimating all unknown parameters based on a MAP estimator, see [24] for more details and advice on bundle adjustment.

Let $m$ denote the number of images and $n$ the number of point features. Denote by $\mathfrak{m}$ the bundle of all unknown parameters, $\mathfrak{m}=\left\{P_{1}, \ldots, P_{m}, \mathbf{X}_{1}, \ldots, \mathbf{X}_{n}\right\}$. Each such element belongs to a non-linear manifold, $\mathcal{M}$. Denote by $\mathfrak{x}=\left(\mathbf{x}_{1}, \ldots, \mathbf{x}_{n}\right)$ all the observable image features. Standard ML estimators optimize

$$
\max _{\mathfrak{m} \in \mathcal{M}} P(\mathfrak{x} \mid \mathfrak{m})
$$

where $P(\mathfrak{x} \mid \mathfrak{m})$ is the probability density function of $\mathfrak{x}$ given $\mathfrak{m}$. If we have information of the bundle, i.e. some probability density function $P(\mathfrak{m})$, this can be incorporated in a MAP estimator,

$$
\max _{\mathfrak{m} \in \mathcal{M}} P(\mathfrak{x} \mid \mathfrak{m}) P(\mathfrak{m}) .
$$

As the probability functions are Gaussian, the problem turns into a non-linear least-squares problem by taking the negative $\log$ of the a posteriori distribution. That is,

$$
\min _{\mathfrak{m} \in \mathcal{M}} Y^{T} Y
$$

where $Y$ is a residual vector consisting of two parts. The first part is formed by computing all reprojection residuals, i.e. the differences between the reprojected 3D points and the measured image points. The residuals should be weighted according to the image measurement accuracy $\sigma_{i m}$. The other part comes from the regularity constraints of the motion parameters.

Introduce a local parameterization $\mathfrak{m}(\Delta \mathbf{x})$, around $\mathfrak{m}_{0} \in$ $\mathcal{M}$ according to

$$
\mathcal{M} \times R^{N} \ni\left(\mathfrak{m}_{0}, \Delta \mathbf{x}\right) \mapsto \mathfrak{m}\left(\mathfrak{m}_{0}, \Delta \mathbf{x}\right) \in \mathcal{M},
$$

where $N=9 m+3 n$. (9 parameters in each camera matrix describing a camera with zero skew and unit aspect ratio and 3 parameters for the coordinates of each reconstructed point.) Let $\Delta \mathbf{x}=\left[\Delta a_{1}, \ldots, \Delta a_{m}, \Delta b_{1}, \ldots, \Delta b_{n}\right]^{T}$, so that $\Delta a_{i}$ parameterize changes in camera matrix $P_{i}$ and $\Delta b_{j}$ parameterize changes in reconstructed point $X_{j}$. Changes in calibration matrix $K_{i}$ are parameterized

$K_{i}\left(\mathfrak{m}_{0}, \Delta \mathbf{x}\right)=\left[\begin{array}{ccc}f+\Delta a_{i}(1) & 0 & x_{0}+\Delta a_{i}(2) \\ 0 & f+\Delta a_{i}(1) & y_{0}+\Delta a_{i}(3) \\ 0 & 0 & 1\end{array}\right]$,

changes in camera centre $\mathbf{C}_{i}$

$$
\mathbf{C}_{i}\left(\mathfrak{m}_{0}, \Delta \mathbf{x}\right)=\left[\begin{array}{l}
\mathbf{C}_{x}+\Delta a_{i}(4) \\
\mathbf{C}_{y}+\Delta a_{i}(5) \\
\mathbf{C}_{z}+\Delta a_{i}(6)
\end{array}\right]
$$

changes in pose $R_{i}$

$$
R_{i}\left(\mathfrak{m}_{0}, \Delta \mathbf{x}\right)=\exp \left(\sum_{k=1}^{3} \Delta a_{i}(6+k) B_{k}\right) R_{i}
$$

where $B_{1}, B_{2}, B_{3}$ is the base for skew symmetric matrices in (4) and finally changes in each object point $\mathbf{X}_{j}$

$$
\mathbf{X}_{j}\left(\mathfrak{m}_{0}, \Delta \mathbf{x}\right)=\left[\begin{array}{c}
X_{j}+\Delta b_{j}(1) \\
Y_{j}+\Delta b_{j}(2) \\
Z_{j}+\Delta b_{j}(3) \\
1
\end{array}\right]
$$

The residuals for the camera centres, cf. (3), focal lengths, cf. (6) and principal points, cf. (7) are $\left(\mathbf{C}_{i}\right.$ $\left.\mathbf{C}_{i+1}\right) / \sigma_{c}, \log \left(f_{i} / f_{i+1}\right) / \sigma_{f}$ and $\left(\left(u_{i}, v_{i}\right)-(\bar{u}, \bar{v})\right) / \sigma_{p p}$, respectively. The residuals for the poses, cf. (5) are obtained by taking the matrix logarithm, as follows

$$
\log \left(R_{i+1} R_{i}^{T}\right)=\sum_{k=1}^{3} \lambda_{k} B_{k}
$$

and weighting the resulting Euler angles with the standard deviation $\sigma_{a}$, resulting in the residuals $\left(\lambda_{1}, \lambda_{2}, \lambda_{3}\right) / \sigma_{a}$.

The residual vector $\mathbf{Y}(\Delta \mathbf{x})$ is a non-linear function of the local parameterization vector $\Delta \mathbf{x}$. In order to minimize the sum of squared residuals $f=\mathbf{Y}^{T} \mathbf{Y}$ with respect to the unknown parameters $\Delta \mathbf{x}$, the Levenberg-Marquardt method is applied.

It is well known that the structure and motion can only be recovered up to an unknown similarity, in particular, the reconstruction is subject to arbitrary scaling. As the camera centre trajectory is modelled as a random walk, the cost function could be decreased by simply reducing the scale. Therefore, we fix the scale by keeping the standard deviation $\sigma_{s c}$ of all 3D point features at its initial value. In practice, we have implemented this by rescaling the reconstruction at each iteration.

\section{Experiments}

In all the different experiments, the same settings have been utilized unless otherwise stated. The global scale has 
been set by fixing the standard deviation of the 3D points (denoted by $\sigma_{s c}$ ) to its initial value. Further, the standard deviations for the random walks have the following values: $\sigma_{i m}=1, \sigma_{c}=\sigma_{s c} / 5, \sigma_{a}=10^{\circ}, \sigma_{f}=0.05$ and $\sigma_{p p}=10$. These values depend of course on the type of scenario and the type of camera. However, the above values are set rather restrictively, making them applicable for a wide range of image sequences.

\subsection{Synthetic Data}

First, 20 points were randomly distributed in 3D space with coordinates between -300 and 300 units. The position of the first camera was chosen at a nominal distance of 1000 units from the origin, facing towards the point cloud. The position $\mathbf{C}_{i}$ of each additional camera centre was updated with $\mathbf{C}_{i}=\mathbf{C}_{i-1}+\mathbf{v}_{i}$, where $\mathbf{v}_{i}$ is a speed vector with a length of 10 units. The direction of $\mathbf{v}_{1}$ was chosen at random. The update formula for the speed was $\mathbf{v}_{i}=\exp \left(\sum_{k=1}^{3} \lambda_{k} B_{k}\right) \mathbf{v}_{i-1}$, where each $\lambda_{k}$ was drawn from a zero-mean Gaussian distribution with standard deviation $10^{\circ}$. The same procedure was also used for the rotation, with a speed vector of length $10^{\circ}$. The focal lengths and principal points were modelled with random walks as described in (6) with $\sigma_{f}=0.05$ and (7) with $\sigma_{p p}=10$. Then, the 3D points were projected to these views, and finally Gaussian noise was added to the image points. The algorithm described in the previous sections, starting from the projective reconstruction step, was applied to this data. All experiments were repeated 100 times and the results below reflect the average values.

In the first experiment, the standard deviation of the image noise was $\sigma=1$ and the number of views was varied. After changing to a common Euclidean coordinate system (based on corresponding 3D points), the Root Mean Square (RMS) error between the estimated and the true camera trajectory was computed for each sample. If the RMS for a sample is over 30 units, it is declared a failure. The RMS error vs. number of views is graphed in the left of Figure 1 for the successful samples. The percentage of failures for the same experiments is plotted in the right of Figure 1. In the second experiment, 10 views were used and the level of image noise was varied. In Figure 2, the RMS error for camera trajectory and percentage of failures are shown.

Even though the synthetic data was not generated according to the a priori noise model for the motion, the experiments show that the performance and robustness increase considerably. This also indicates that exact values of the parameters in the random walk are not crucial to increase performance. It is well known that there exist many local minima for auto-calibration and the structure and motion problem. Since the number of outliers is fewer with regularization, it is likely that the bundle adjustment does not
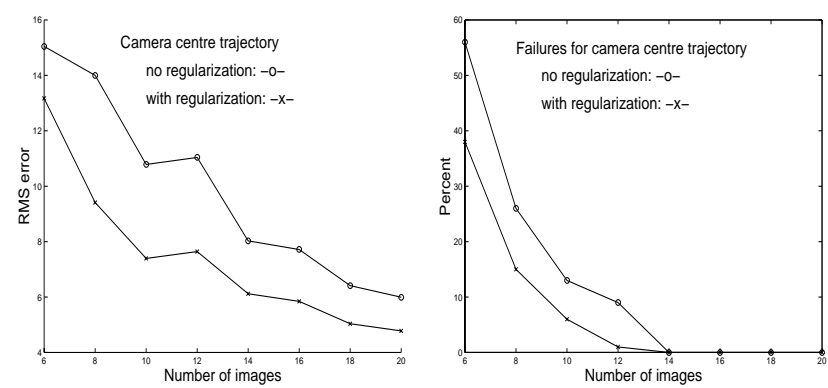

Figure 1. Varying number of images. Left: RMS error of camera trajectory. Right. Percentage of failures.
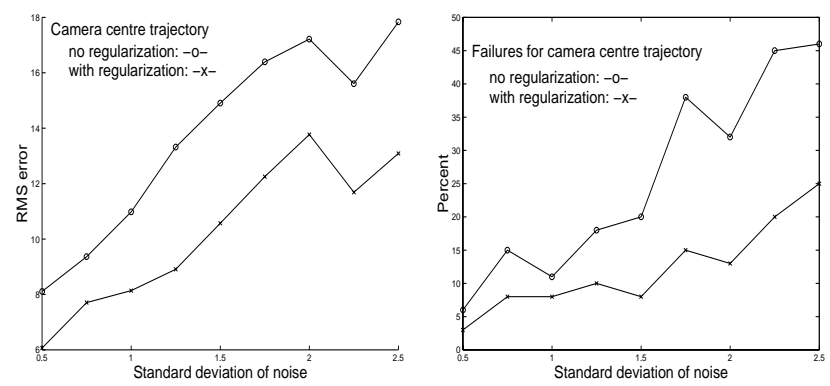

Figure 2. Varying levels of noise. Left: RMS error of camera trajectory Right: Percentage of failures.

get trapped so easily in a false minimum. This is of course desirable and one of the motivations for modelling the motion.

\subsection{Real Data}

We have chosen two image sequences to show the performance of using regularity conditions on the motion compared to standard bundle adjustment.

The first sequence consists of 15 closely sampled images of a flower, see Figure 3 for an example. The images have been taken with an ordinary digital camera with a resolution of $1000 \times 1280$ pixels. Between the imaging instants, the zoom of the camera has been varied, but in a controlled way. The first 5 images have a (relative) short focal length which is kept fixed during the subsequence. The next 5 images have also a fixed focal length, but the camera has zoomed in and thus it should have a longer focal length. In the last 5 images the camera has zoomed in even more, and it should have a longer focal length, but again it should be constant during the 5 images. However, no other ground 


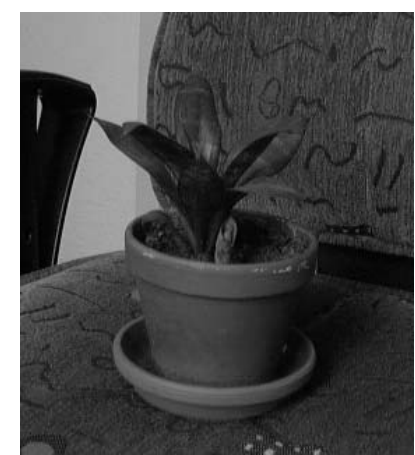

Figure 3. One image in the flower sequence.
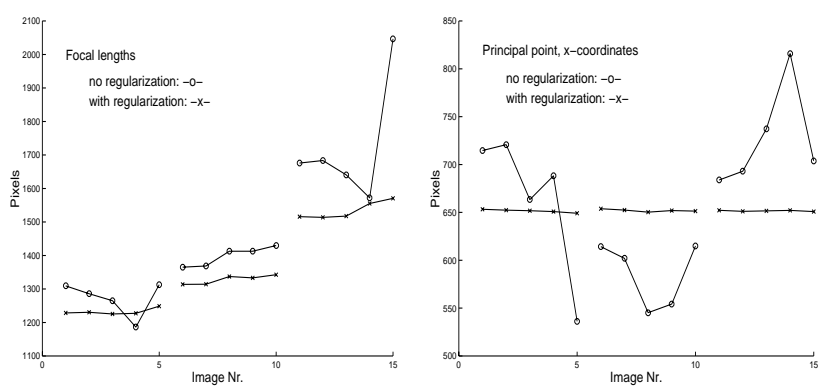

Figure 4. Flower sequence. Left: Focal lengths. Right: $X$-coordinates of principal point.

truth is available. In total 41 points were tracked over the sequence, where some of the points are not visible in all images.

After applying the complete algorithm with and without regularity conditions, the RMS error between measured and reprojected 3D points was computed. Without regularity, the RMS error was 0.15 pixels and with regularity, the error was as could be expected somewhat higher 0.18 pixels. The computed focal lengths are plotted in the left of Figure 4. As can be clearly seen, with regularity conditions the focal lengths are approximately constant for the 3 subsequences of 5 images, and the focal lengths are increasing. Without regularity conditions, the algorithm produces focal lengths that are much more noisy and the focal lengths are not constant within the 3 subsequences.

The difference between the two results are even more accentuated in the right graph of Figure 4 where the $x$ coordinates of the principal point are graphed. The principal point is known to vary when a camera zooms, but the variations are small. This is in agreement with the MAP estimator modelling the motion, whereas the standard bundle adjustment fails to recover a reasonable estimate of the
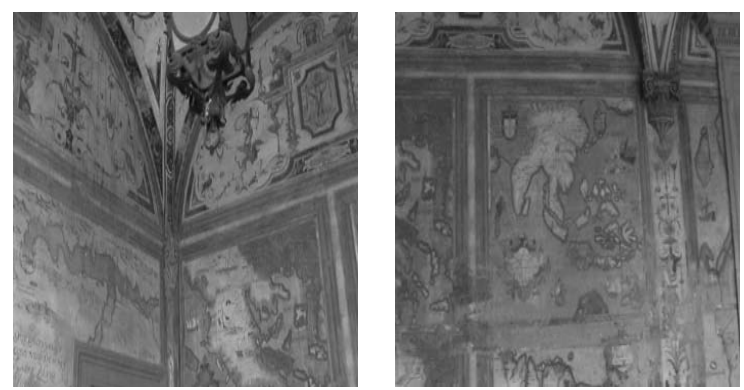

Figure 5. A room in Villa Laloggia with hand painted walls and ceiling. The 6th and the last images of the sequence are shown.

principal point.

The second sequence of images that we demonstrate our algorithm on consists of 16 images taken by a digital video camera. The images originate from a room with hand painted walls and ceiling in Villa Laloggia, which is a wellknown historical building in Florence, Italy, see Figure 5. The intrinsic camera parameters are known to be kept constant over the whole sequence. This a priori knowledge has been incorporated in the algorithm by parameterizing the intrinsic parameters by constant values. The sequence covers only part of the room, primarily two walls, a lamp and parts of the ceiling. More interestingly, the last image of the sequence covers only one of the walls, cf. Figure 5 and thus all visible features lie on a common 3D plane. This is well-known to be a degenerate configuration for projective reconstruction. In theory, auto-calibration is still possible, but it is more unstable [23].

In total 861 points were tracked and reconstructed in 3D. On the average 250 points were visible in each image. First, the algorithm without regularity conditions, parameterized by constant intrinsics, was applied to the sequence. The RMS error for the whole sequence was 0.67 pixels and for the last image 0.36 pixels. A $3 \mathrm{D}$ plot of the camera trajectory and the 3D points is shown on the left side of Figure 6. At a first glance everything seems to be $\mathrm{OK}$, but it is not. All camera centres but the last one are situated on the inside of the room. However, the position of the last camera centre is on the other side of the wall. This is due to the fact that the camera faces a planar wall and therefore there are two possible positions (one in front, and the other on the opposite side) that are consistent with the image data. The situation would not be resolved by using visibility constraints, as all points are indeed in front of all cameras.

Using regularity conditions for the motion, there is only one side of the wall that is consistent. A RMS error of 0.77 pixels and 0.36 pixels were obtained for the whole sequence and for the last image, respectively, after applying the algo- 

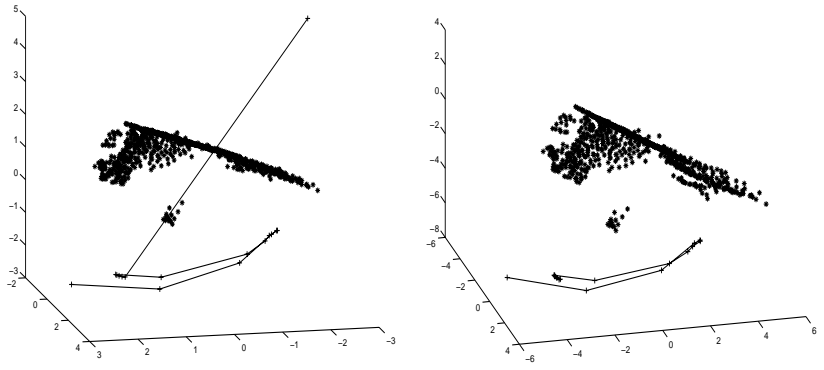

Figure 6. 3D plot of trajectory of camera centres (crosses) and reconstructed 3D points (stars) for Villa Laloggia sequence. Left: Without regularization. Right: With regularization.

rithm with regularization. A 3D plot of the camera trajectory and reconstructed 3D points is shown on right side of Figure 6. Now, the position of the last camera is correctly situated on the inside of the room.

\section{Summary and Conclusions}

In this paper, we have described how to model smoothness constraints on the camera motion, and how to incorporate these in an MAP estimator for Euclidean structure and motion. The experiments show that the proposed algorithm yield more accurate and robust results compared to traditional bundle adjustment. In particular, the regularity constraints reduce the risk of some well-known failure modes for conventional bundle adjustment, like (i) critical motions leading to ambiguous 3D reconstructions, (ii) high correlation between the extrinsic and intrinsic parameters of the camera and (iii) the risk of getting trapped in a local minimum.

\section{References}

[1] M. Armstrong, A. Zisserman, and R. Hartley. Self-calibration from image triplets. In European Conf. Computer Vision, pages 3-16, Cambridge, UK, 1996.

[2] K. Åström and A. Heyden. Euclidean reconstruction from constant intrinsic parameters. In Int. Conf. Pattern Recognition, volume I, pages 339-343, Vienna, Austria, 1996.

[3] A. Azarbayejani and A. Pentland. Recursive estimation of motion, structure, and focal length. IEEE Trans. Pattern Analysis and Machine Intelligence, 17(6):545-561, 1995.

[4] T. Brodsky, C. Fermuller, and Y. Aloimonos. Self-calibration from image derivatives. In Int. Conf. Computer Vision, pages 83-89, Mumbai, India, 1998.

[5] O. Faugeras, Q.-T. Luong, and S. Maybank. Camera self-calibration: Theory and experiments. In European Conf. Computer Vision, pages 321-334, Santa Margherita Liguere, Italy, 1992. Springer-Verlag.
[6] A. Fitzgibbon and A. Zisserman. Automatic camera recovery for closed or open image sequences. In European Conf. Computer Vision, volume I, pages 311-326, Freiburg, Germany, 1998.

[7] R. I. Hartley. Euclidean reconstruction from uncalibrated views. In J. L. Mundy and A. Zisserman, editors, Applications of Invariance in Computer Vision, pages 237-256. Springer-Verlag, Berlin, Heidelberg, 1994.

[8] R. I. Hartley and A. Zisserman. Multiple View Geometry in Computer Vision. Cambridge University Press, 2000.

[9] A. Heyden and K. Åström. Euclidean reconstruction from image sequences with varying and unknown focal length and principal point. In Conf. Computer Vision and Pattern Recognition, pages 438-443, San Juan, Puerto Rico, 1997.

[10] A. Heyden and K. Åström. Flexible calibration: Minimal cases for auto-calibration. In Int. Conf. Computer Vision, pages 350-355, Kerkyra, Greece, 1999.

[11] F. Kahl, B. Triggs, and K. Åström. Critical motions for autocalibration when some intrinsic parameters can vary. Journal of Mathematical Imaging and Vision, 13(2):131-146, 2000.

[12] Q.-T. Luong and O. Faugeras. Self-calibration of a moving camera from point correspondances and fundamental matrices. Int. Journal Computer Vision, 22(3):261-289, 1997.

[13] A. Manessis, A. Hilton, P. Palmer, P. McLauchlan, and X. Shen. Reconstruction of scene models from sparse $3 \mathrm{~d}$ structure. In Conf. Computer Vision and Pattern Recognition, volume 2, pages 666-671, Hilton Head SC, USA, 2000.

[14] P. McLauchlan. A batch/recursive algorithm for $3 \mathrm{~d}$ scene reconstruction. In Conf. Computer Vision and Pattern Recognition, volume 2, pages 738-743, Hilton Head SC, USA, 2000.

[15] D. Morris and T. Kanade. Image-consistent surface triangulation. In Conf. Computer Vision and Pattern Recognition, volume 1, pages 332-338, Hilton Head SC, USA, 2000.

[16] M. Pollefeys, R. Koch, and L. Van Gool. Self-calibration and metric reconstruction in spite of varying and unknown internal camera parameters. In Int. Conf. Computer Vision, pages 90-95, Mumbai, India, 1998.

[17] M. Pollefeys, L. Van Gool, and M. Proesmans. Euclidean 3d reconstruction from image sequences with variable focal lengths. In European Conf. Computer Vision, pages 31-42, Cambridge, UK, 1996.

[18] S. Soatto, R. Frezza, and P. Perona. Motion estimation via dynamic vision. IEEE Trans. Automatic Control, 41(3):393-413, 1996.

[19] F. Spitzer. Principles of Random Walk. Van Nostrand Company, 1964

[20] P. Sturm. Critical motion sequences for monocular self-calibration and uncalibrated Euclidean reconstruction. In Conf. Computer Vision and Pattern Recognition, pages 1100-1105, San Juan, Puerto Rico, 1997

[21] P. Torr and A. Zisserman. Robust computation and parametrization of multiple view relations. In Int. Conf. Computer Vision, pages 727732, Mumbai, India, 1998.

[22] B. Triggs. Autocalibration and the absolute quadric. In Conf. Computer Vision and Pattern Recognition, pages 609-614, San Juan, Puerto Rico, 1997.

[23] B. Triggs. Autocalibration from planar scenes. In European Conf. Computer Vision, volume I, pages 89-105, Freiburg, Germany, 1998.

[24] B. Triggs, P. McLauchlan, Hartley R., and A. Fitzgibbon. Bundle adjustment - a modern synthesis. In Vision Algorithms'99, in conjunction with ICCV'99, Kerkyra, Greece, 1999.

[25] J. Weng, N. Ahuja, and T.S. Huang. Optimal motion and structure estimation. IEEE Trans. Pattern Analysis and Machine Intelligence, 15(9):864-884, 1993. 